\title{
NEWS
}

\section{Measles resurgence comes to Canada}

\author{
Cite as: CMAJ 2019 March 18;191:E319. doi: 10.1503/cmaj.109-5724
}

Posted on cmajnews.com on Feb. 26, 2019.

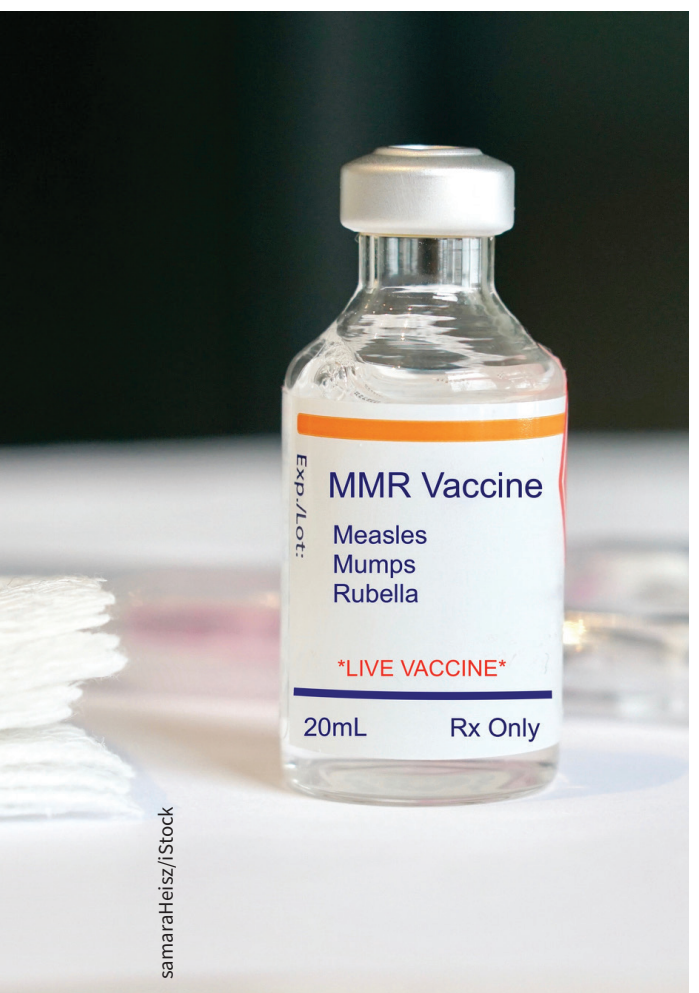

Vaccine hesitancy is a contributing factor to measles outbreaks around the world.

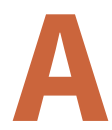

$\mathrm{n}$ outbreak of measles in Canada is what public health officials say they have been fearing. The most likely scenario for an outbreak is an unvaccinated visitor from an endemic area, or an unvaccinated Canadian returning from such an area, bringing in the virus and spreading it among contacts.

That appears to be what happened in Vancouver, where a recent outbreak started with an unvaccinated 11-year-old boy returning from a family trip to Vietnam. His parents had reportedly not vaccinated him because of concerns about the safety of the measles, mumps and rubella (MMR) vaccine.
"The elephant in the room is 'vaccine hesitancy' - parents who are on the fence or not quite sure about vaccination because all of the information and misinformation out there," said Dr. Howard Njoo, deputy chief public health officer at the Public Health Agency of Canada (PHAC). "Our worry - and I think I speak for a lot of public health officials - is that younger generations have not experienced measles" and fail to realize how serious it can be.

Dr. Sarah Wilson of Public Health Ontario sees other gaps in vaccine coverage. Imported cases tend to be in infants less than a year old who have travelled to endemic countries. "Their parents are often unaware that the child could get a first dose of MMR vaccine at 6 months." She said other cases are in adults, who may think their measles immunization is up to date but may not have had the two doses required.

Such gaps in coverage can allow measles to spread, as an immunization rate of $95 \%$ is needed to provide herd immunity to such an easily transmitted disease, according to the World Health Organization (WHO). Njoo says Canada's immunization rate, at $89 \%$, is "not bad, but we've got a way to go." He is concerned about pockets of the population who are under-vaccinated owing to religious beliefs. "We've seen that these groups get together with members of their faith from another country. This has led to outbreaks in the past."

Endemic countries now include all of Europe, where the WHO says more than 84000 people in 47 countries had measles in 2018, with two-thirds winding up in hospital and 72 dying. Cases are up 15-fold over 2016, when they were at a record low.

Countries around the globe such as Israel, Ukraine and the Philippines are reporting outbreaks. The United States reported 127 cases in the first six weeks of 2019 , putting it on track to exceed the spike of 667 cases recorded in 2014. The largest US outbreaks are in New York and Washington state.

Guarding against such outbreaks puts Canada's doctors on the frontline for prevention. PHAC provides disease surveillance and information for health care professionals, but Njoo stressed that physicians can also deliver a personal message. He tells patients, "I've seen the benefits of vaccination. I've had my children vaccinated."

Physicians are also responsible for diagnosing measles, which is not always straightforward.

"Local public health units send out health care provider communications about presenting signs and symptoms," said Wilson. "But in the very early stages, symptoms may not be specific. Certainly, with our cases with a recent history of exposure, they go to a walk-in clinic, then another one, and then an emergency department, which presents challenges in terms of control." She said measles should be considered "especially if patients have a travel history in areas with very high-profile outbreaks or time spent in an international airport."

Even before the measles rash appears, "with the runny nose, conjunctivitis, and classic Koplik's spots in the mouth, I would hope most physicians would catch it," said Njoo.

"We would never be complacent about measles control," said Wilson. "We are watching very closely any case of measles importation. There's a 21-day incubation period, and we take a deep breath after the period is over."

Carolyn Brown, Ottawa, Ont. 